\title{
Pathological analysis of the superior mesenteric artery boundary in preoperative computed tomography of resectable pancreatic head adenocarcinoma
}

\author{
MENG LU ${ }^{1 *}$, CHUN-HUI YUAN ${ }^{*}$, LING-FU ZHANG ${ }^{1}$, LIAN-YUAN TAO ${ }^{1}$, \\ YING PENG $^{1}$, LI-MEI GUO ${ }^{2}$, GANG LI ${ }^{1}$ and DIAN-RONG XIU ${ }^{1}$ \\ Departments of ${ }^{1}$ General Surgery and ${ }^{2}$ Pathology, Peking University Third Hospital, Beijing 100191, P.R. China
}

Received May 13, 2018; Accepted March 26, 2019

DOI: $10.3892 / 01.2019 .10269$

\begin{abstract}
The aim of the present study was to evaluate the biological and prognostic implications of the superior mesenteric artery (SMA) boundary on preoperative abdominal contrast-enhanced computed tomography (CE-CT) for resectable adenocarcinoma of the pancreatic head. A total of 121 patients treated over a 6-year period at Peking University Third Hospital (Beijing, China) were included in the present study. The pattern of the SMA boundary was investigated on preoperative CE-CT and detailed pathological analysis of the extrapancreatic plexus [the pancreatic head plexus II (PLX-II) located on the right edge of the SMA] was performed. The results revealed that the radiological SMA boundary was associated with the grade of parasympathetic neurogenesis $(\mathrm{P}=0.014)$ in $\mathrm{PLX}-\mathrm{II}$, and was predictive of postoperative disease-free survival $(\mathrm{P}=0.014)$ and liver metastasis $(\mathrm{P}=0.013)$. Therefore, it was proposed that extrapancreatic parasympathetic neurogenesis may account for the different patterns of the SMA boundary on preoperative abdominal CE-CT, and
\end{abstract}

Correspondence to: Dr Dian-Rong Xiu, Department of General Surgery, Peking University Third Hospital, 49 North Garden Road, Haidian, Beijing 100191, P.R. China

E-mail: xiudianrong@163.com

${ }^{*}$ Contributed equally

Abbreviations: CE-CT, contrast-enhanced computed tomography; SMA, superior mesenteric artery; PLX-II, pancreatic head plexus II; SMV, superior mesenteric vein; PV, portal vein; VAChT, vesicular acetylcholine transporter; PBS, phosphate-buffered saline; UICC, Union for International Cancer Control; OS, overall survival; DFS, disease-free survival; LR, locoregional recurrence; LM, liver metastasis; CA19-9, cancer antigen 19-9; TBIL, total bilirubin; IPNI, intrapancreatic neural invasion; EPNI, extrapancreatic neural invasion; ASCO, American Society of Clinical Oncology

Key words: resectable pancreatic head adenocarcinoma, superior mesenteric artery, extrapancreatic neuropathy, parasympathetic neurogenesis, liver metastasis affect the prognosis, particularly for liver metastasis in resectable pancreatic head adenocarcinoma.

\section{Introduction}

Pancreatic ductal adenocarcinoma is a one of the most aggressive types of cancer in the digestive system and is associated with a poor prognosis, with a 5-year survival rate of $<6 \%(1,2)$. Radical resection is currently the best treatment option for curative outcome and long-term survival rates, however, only $15-20 \%$ of patients are suitable candidates for curative resection as first-line treatment at initial diagnosis (3). Some distant micrometastases are undetectable at first diagnosis, despite thorough preoperative imaging tests, and may only be confirmed by examination during planned curative resection or by rapid relapse shortly following surgery $(1,3)$. For this reason, patients undergoing curative pancreaticoduodenectomy have a $25-50 \%$ risk of recurring local or distant metastases, and those with metastatic disease have a median life expectancy of $~ 3-6$ months and a 5-year relative survival rate of only $1 \%(1,3,4)$. Therefore, efforts to identify factors that predict early recurrence in patients with pancreatic ductal adenocarcinoma undergoing surgical resection are warranted.

The pancreatic head is the most common location for the development of pancreatic ductal adenocarcinoma, and resectable pancreatic head adenocarcinoma does not involve the main peripancreatic arteries (5). Previous studies have described tumor resectability in pancreatic ductal adenocarcinoma, however, the majority of definitions of resectable pancreatic head adenocarcinoma are based on the technical or anatomical resectability of the tumor during preoperative abdominal contrast-enhanced computed tomography (CE-CT), with little focus on the potential biological behavior of the tumor (6). Consequently, patients with radiologically diagnosed resectable pancreatic cancer frequently have a poor prognosis, even following radical surgery $(5,6)$.

The superior mesenteric artery (SMA) is an important anatomic structure for predicting prognosis in patients with pancreatic head adenocarcinoma (7). Tumor involvement of the SMA is defined as one of the criteria for diagnosing borderline resectable pancreatic cancer in various guidelines $(6,8-10)$. The SMA margin is one of the incised margins in pancreatic 
head adenocarcinoma, and is described as the soft tissues directly adjacent to the proximal 3-4 cm of the SMA, mainly containing the pancreatic head plexus II (PLX-II) and other tissues including fibrous and adipose tissues, lymphatic vessels and capillaries, all of which communicate with the uncinate process $(7,11,12)$. The SMA margin is the surgical margin with the highest frequency of residual tumor, which leads to early recurrence following tumor resection $(11,13)$. On preoperative CE-CT, the SMA margin or the PLX-II is approximately equal to the right side of the SMA boundary, although the patterns of the SMA boundary on preoperative CE-CT may vary among patients $(14,15)$. In clinical experience, patients with obscure SMA boundaries often present with early recurrence following radical resection. Therefore, the aim of the present retrospective study was to evaluate the biological and prognostic implications of the SMA boundary on preoperative CE-CT, and investigate the characteristics of extrapancreatic neuropathy.

\section{Patients and methods}

Patient recruitment. The present study was approved by the Institutional Review Board of the Peking University Third Hospital (Beijing, China). Patients with pancreatic head cancer who underwent radical surgery at the Department of General Surgery of the Peking University Third Hospital between January 2010 and December 2015 were included. All patients were radiologically diagnosed with resectable pancreatic head cancer, without invasion of the main peripancreatic arteries, and were all treated with surgery. Postoperative pathological analysis of the tumor confirmed the diagnosis of pancreatic ductal adenocarcinoma. No distant metastases were identified prior to or during surgery, and all fatalities were due to tumor recurrence. The clinicopathological characteristics and postoperative outcomes of these patients were recorded. The observation period was between January 2010 and December 2017.

Patterns of the SMA boundary. Abdominal CE-CT was preoperatively performed with a 64-detector row scanner (Siemens AG, Munich, Germany; Definition 2008 G H-SP) in all patients. The technical parameters were standardized as follows: $120 \mathrm{kV}, 36 \mathrm{~mA}$ and 3-mm-thick contiguous sections. The CE-CT scans were retrospectively analyzed by three experienced gastroenterologists (DX, CY and YP), who all had at least 10 years of clinical experience with abdominal CE-CT as part of their daily clinical and research practice. Results identified by more than one gastroenterologist were considered the final results for each patient. All images were reviewed on a Picture Archiving and Communications System workstation monitor (General Electric, Co., Ltd.). The reviewers were aware that the patients had undergone radical surgery for pancreatic cancer, although they were blinded to all surgical and pathological findings. All phases of the CE-CT scans were evaluated as a whole.

'Tumor invasion' of the SMA referred to arterial-tumor direct contact, which was confirmed in the arterial phase on preoperative CE-CT; patients with true SMA invasion were excluded from the present study. The pathway of the SMA in the arterial phase of preoperative CE-CT was examined, mainly focusing on the region located between the SMA and the uncinate process. The presence of soft tissues in this region was defined as 'all or none', and it was suggested that the normal soft tissue around the SMA may be distinguished from the tumor invasion in this manner. The characteristics of the tissues in this region differed among patients. If the tissues in this region exhibited fat density on imaging, then the SMA boundary was defined as clear. If confluent soft tissue replaced the adipose tissue in this region, the SMA boundary was defined as obscure (Fig. 1). All patients were classified into these two groups according to the patterns of the SMA boundary.

Tissue processing. All patients underwent standard pancreaticoduodenectomy according to tumor location and lymph node metastasis. The Kocher maneuver (16) was performed to expose the junction of the left renal vein and the inferior vena cava, as it helped to confirm the position of the SMA. The pancreatic neck was dissected in front of the superior mesenteric vein (SMV). Following gentle pushing of the SMV to the inner side using a vascular retractor, the tissues in the SMA margin were exposed, followed by right-sided semi-circumferential dissection of the PLX-II, which is the plexus surrounding the SMA (Fig. 2). The SMV or portal vein (PV) was partially resected and reconstructed, either by end-to-end anastomosis or by insertion of a venous graft, if tumor infiltration was identified.

Immunohistochemical staining. The PLX-II tissues in the SMA margin were pathologically examined individually. Five paraffin-embedded $3-\mu \mathrm{m}$ sections of PLX-II tissues were obtained from each patient, and immunohistochemical staining with a rabbit polyclonal antibody against the vesicular acetylcholine transporter (VAChT; 1:100; cat. no. ab-68984; Abcam, Cambridge, MA, USA) was performed in each section to observe the parasympathetic nerves in the SMA margin. Briefly, all sections were deparaffinized with xylene and rehydrated, and antigen retrieval was achieved via heat treatment in an autoclave in $10 \mathrm{mmol} / \mathrm{l}$ sodium citrate buffer (pH 6.0) for $30 \mathrm{~min}$. All sections were further treated with methanol and $3 \%$ hydrogen peroxide prior to washing with phosphate-buffered saline (PBS). Bovine serum albumin (5\%; cat. no. A8850-5; Beijing Solarbio Science \& Technology Co., Ltd., Beijing, China) was used for $1 \mathrm{~h}$ at room temperature to block background staining, following which the membrane was incubated overnight with the primary antibody at $4^{\circ} \mathrm{C}$. The detection of the antigen-antibody complex was performed using the Super Sensitive ${ }^{\mathrm{TM}}$ Polymer-HRP IHC Detection system (BioGenex Laboratories, Fremont, CA, USA), according to the manufacturer's protocol. PBS was used instead of a primary antibody for the negative control (17). One pathologist, who was blinded to the clinical outcome, independently scored the results of the staining.

Pathological analysis. The surgical margins were considered to be microscopically positive (R1) if carcinoma was present at the transection lines or in the dissected peripancreatic soft tissue margins within $1 \mathrm{~mm}$. R0 resection was defined as no microscopic evidence of cancer cells along all margins (18). The final stage of pancreatic head adenocarcinoma was determined 

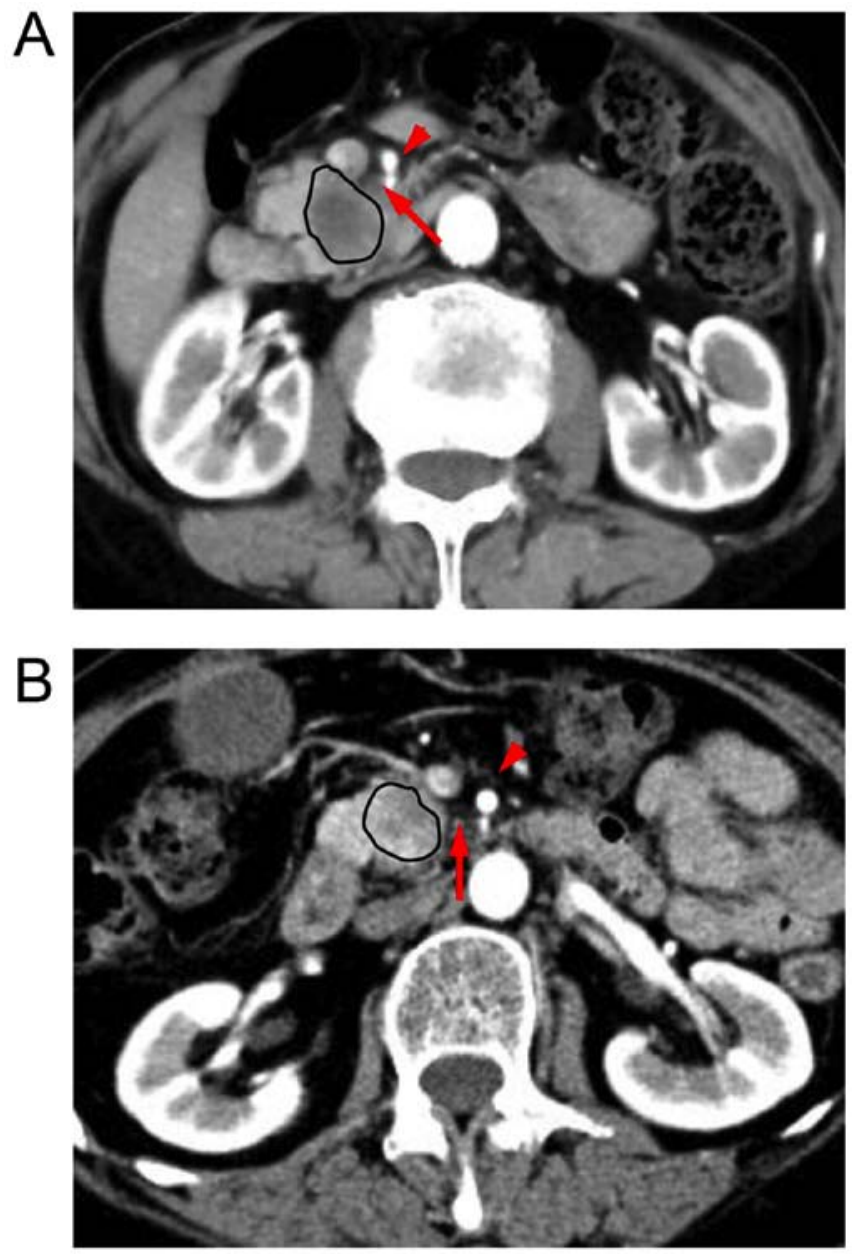

Figure 1. Patterns of the SMA boundary in the arterial phase on preoperative abdominal contrast-enhanced computed tomography. The short red arrows indicate the SMA, the long red arrows indicate tissues in the SMA margin, and the black circles indicate pancreatic head adenocarcinoma. (A) Obscure SMA boundary. (B) Clear SMA boundary. SMA, superior mesenteric artery.

pathologically according to the tumor-node-metastasis classification system of malignant tumors published by the Union for International Cancer Control (UICC), 8th edition (19).

Parasympathetic neurogenesis is a morphological phenomenon in the tumor microenvironment, involving the distribution of abnormal parasympathetic nerve fibers in the tumor stroma. Nerve fibers of various shapes and other constituents, including adipose and fibrous tissue, were evaluated. Parasympathetic neurogenesis was assessed by immunohistochemical staining of tissue sections. The number of parasympathetic nerve fibers was calculated in each section, and the mean number of five sections from each patient was compared among the different groups.

Follow-up observations. All patients were followed up by physical examination and abdominal CE-CT every 3-6 months. The follow-up time was defined as the time from the date of the first surgery to the date of the final follow-up, and was recorded in months. Follow-up information was obtained from office charts, hospital records and telephone interviews. Overall survival (OS) was defined as the time from the date of the first surgery to the date of patient mortality due to recurrence. Disease-free survival (DFS) was defined as the time from the date of the first surgery to the date of the first evidence of recurrence [loco-regional recurrence (LR) or distant metastasis]. Early recurrence was defined as relapse within 12 months. The patterns of recurrence sites were subdivided into LR and liver metastasis (LM); the former indicated a recurrence presumably due to occult residual tumor cells left behind at the time of resection, whereas the latter is the most common cause of mortality among patients with pancreatic ductal adenocarcinoma who undergo radical surgery.

Statistical analysis. All statistical analyses were conducted using SPSS software (version 23.0 for Windows; IBM Corp., Armonk, NY, USA). Correlation analyses among various groups and different biological factors were performed using the $\chi^{2}$ test and independent sample t-tests. Survival analysis was performed by constructing Kaplan-Meier curves for OS, DFS, LR and LM. The log-rank test was used to compare survival differences among the groups. Multivariate Cox regression models (backward stepwise likelihood ratio) were used to analyze factors affecting survival rate, and reported with a hazard ratio of 1.0 as the baseline and $95 \%$ confidence intervals. $\mathrm{P}<0.05$ was considered to indicate a statistically significant difference.

\section{Results}

Clinicopathological characteristics. According to the inclusion and exclusion criteria, 121 patients who underwent radical tumor resection were included in the present study. A total of 67 men and 54 women were included, ranging in age between 23 and 84 years, with a mean age of 62.0 years. All diagnoses were ultimately confirmed clinically and pathologically. Of the 121 patients, $68(56.2 \%)$ had clear SMA boundaries on preoperative CE-CT, whereas 53 (43.8\%) had obscure boundaries. Increased cancer antigen $19-9\left(\mathrm{CA}_{19-9}\right)$ levels were observed in $83.5 \%(101 / 121)$ of the patients, and increased total bilirubin (TBIL) levels were found in $72.7 \%(88 / 121)$ of the patients. In $46(38.0 \%)$ patients, venous (SMV and PV) invasion was identified during surgery, and 20 (16.5\%) patients received venous resection and reconstruction. Lymph node invasion was observed in 65 patients (53.7\%), among whom $51(42.1 \%)$ were classified as N1 stage and $14(11.6 \%)$ were classified as N2 stage patients. Based on the most recent UICC classification system, 48 (39.7\%) cases were defined as stage III carcinoma. R0 resections were achieved in 58 (47.9\%) patients, and a positive SMA margin (R1) was observed in 36 $(29.8 \%)$ patients. Cancer emboli in the vessels were observed in $35(28.9 \%)$ patients, and intrapancreatic neural invasion (IPNI) was identified in $90(74.4 \%)$ patients. In addition, the carcinoma was poorly differentiated in $54.5 \%(66 / 121)$ of the cases. Within 12 months, 63.6\% (77/121) of the patients had developed recurrence and $47.1 \%$ (57/121) succumbed to the disease. Of the patients with early recurrence, 57 (47.1\%) had LM and 32 (26.4\%) had LR. All other characteristics are summarized in Table I.

The clinicopathological parameters were examined in the two groups (Table I). There was no correlation between the pattern of the SMA boundary and age $(\mathrm{P}=0.934)$, gender $(\mathrm{P}=0.898)$, preoperative levels of $\mathrm{CA}_{19-9}(\mathrm{P}=0.173)$ or $\mathrm{TBIL}$ $(\mathrm{P}=0.067)$, venous resection $(\mathrm{P}=0.541)$ or adjuvant therapy 
Table I. Clinical and pathological characteristics of 121 patients.

\begin{tabular}{|c|c|c|c|c|c|}
\hline Characteristic & Variable & $\mathrm{N}(\%)$ & $\begin{array}{c}\text { Clear SMA } \\
\text { boundary }(n=68)\end{array}$ & $\begin{array}{c}\text { Obscure SMA } \\
\text { boundary }(n=53)\end{array}$ & P-value \\
\hline \multicolumn{6}{|l|}{ Clinical characteristic } \\
\hline \multirow[t]{3}{*}{ Age (years) } & & & & & $0.934^{\mathrm{a}}$ \\
\hline & $\leq 65$ & $69(57.0)$ & $39(57.4)$ & $30(56.6)$ & \\
\hline & $>65$ & $52(43.0)$ & $29(42.6)$ & $23(43.4)$ & \\
\hline \multirow[t]{3}{*}{ Gender } & & & & & $0.898^{\mathrm{a}}$ \\
\hline & Male & $67(55.4)$ & $38(55.9)$ & $29(54.7)$ & \\
\hline & Female & $54(44.6)$ & $30(44.1)$ & $24(45.3)$ & \\
\hline \multirow[t]{3}{*}{$\mathrm{CA}_{19-9}(\mathrm{U} / \mathrm{ml})$} & & & & & $0.173^{\mathrm{a}}$ \\
\hline & $\leq 39$ & $20(16.5)$ & $14(20.6)$ & $6(11.3)$ & \\
\hline & $>39$ & $101(83.5)$ & $54(79.4)$ & $47(88.7)$ & \\
\hline \multirow[t]{3}{*}{ TBIL $(\mu \mathrm{mol} / \mathrm{L})$} & & & & & $0.067^{\mathrm{a}}$ \\
\hline & $\leq 17.1$ & $33(27.3)$ & $23(33.8)$ & $10(18.9)$ & \\
\hline & $>17.1$ & $88(72.7)$ & $45(66.2)$ & $43(81.1)$ & \\
\hline Venous invasion & & $46(38.0)$ & $19(27.9)$ & $27(50.9)$ & $0.010^{\mathrm{a}}$ \\
\hline Venous resection & & $20(16.5)$ & $10(14.7)$ & $10(18.9)$ & $0.541^{\mathrm{a}}$ \\
\hline Adjuvant therapy & & $56(46.3)$ & $32(47.1)$ & $24(45.3)$ & $0.846^{\mathrm{a}}$ \\
\hline Early mortality & & $57(47.1)$ & $24(35.3)$ & $33(62.3)$ & $0.003^{\mathrm{a}}$ \\
\hline Early recurrence & & 77 (63.6) & $34(50.0)$ & $43(81.1)$ & $<0.001^{\mathrm{a}}$ \\
\hline Early LM & & $57(47.1)$ & $23(33.8)$ & $34(64.2)$ & $0.001^{\mathrm{a}}$ \\
\hline Early LR & & $32(26.4)$ & $14(20.6)$ & $18(34.0)$ & $0.098^{\mathrm{a}}$ \\
\hline Median OS time (months) & & & $24.9 \pm 3.6$ & $13.9 \pm 1.7$ & $0.005^{\mathrm{b}}$ \\
\hline Median DFS time (months) & & & $22.1 \pm 3.6$ & $9.0 \pm 1.1$ & $0.003^{b}$ \\
\hline Median time without LR (months) & & & $40.1 \pm 5.6$ & $23.6 \pm 3.6$ & $0.182^{b}$ \\
\hline Median time without LM (months) & & & $36.5 \pm 4.6$ & $11.2 \pm 1.5$ & $0.001^{\mathrm{b}}$ \\
\hline \multicolumn{6}{|l|}{ Pathological characteristics } \\
\hline \multirow[t]{3}{*}{ Surgical margin } & & & & & $0.212^{\mathrm{a}}$ \\
\hline & R0 & $58(47.9)$ & $36(52.9)$ & $22(41.5)$ & \\
\hline & $\mathrm{R} 1$ & $63(52.1)$ & $32(47.1)$ & $31(58.5)$ & \\
\hline \multirow[t]{3}{*}{ SMA margin } & & & & & $<0.001^{\mathrm{a}}$ \\
\hline & R0 & $85(70.2)$ & $57(83.8)$ & $28(52.8)$ & \\
\hline & $\mathrm{R} 1$ & $36(29.8)$ & $11(16.2)$ & $25(47.2)$ & \\
\hline Intrapancreatic neural invasion & & $90(74.4)$ & $48(70.6)$ & $42(79.2)$ & $0.279^{\mathrm{a}}$ \\
\hline Cancer embolus in vessel & & 35 (28.9) & $25(36.8)$ & $10(18.9)$ & $0.031^{\mathrm{a}}$ \\
\hline Poor differentiation & & $66(54.5)$ & $37(54.4)$ & $29(54.7)$ & $0.973^{\mathrm{a}}$ \\
\hline \multirow[t]{5}{*}{ T stage } & & & & & $<0.001^{\mathrm{a}}$ \\
\hline & $\mathrm{T} 1$ & $4(3.3)$ & $4(5.9)$ & $0(0)$ & \\
\hline & $\mathrm{T} 2$ & $65(53.7)$ & $48(70.6)$ & $17(32.1)$ & \\
\hline & $\mathrm{T} 3$ & $12(9.9)$ & $8(11.8)$ & $4(7.5)$ & \\
\hline & $\mathrm{T} 4$ & $40(33.1)$ & $8(11.8)$ & $32(60.4)$ & \\
\hline \multirow[t]{4}{*}{ N stage } & & & & & $0.556^{\mathrm{a}}$ \\
\hline & N0 & $56(46.3)$ & $32(47.1)$ & $24(45.3)$ & \\
\hline & N1 & $51(42.1)$ & $30(44.1)$ & $21(39.6)$ & \\
\hline & N2 & 14 (11.6) & $6(8.8)$ & $8(15.1)$ & \\
\hline \multirow[t]{4}{*}{ UICC stage } & & & & & $<0.001^{\mathrm{a}}$ \\
\hline & I & $30(24.8)$ & $24(35.3)$ & $6(11.3)$ & \\
\hline & II & $43(35.5)$ & $31(45.6)$ & $12(22.6)$ & \\
\hline & III & $48(39.7)$ & $13(19.1)$ & $35(66.0)$ & \\
\hline Size of largest tumor $(\mathrm{cm})$ & & $3.1 \pm 1.9$ & $3.4 \pm 2.5$ & $3.3 \pm 1.4$ & $0.831^{\mathrm{c}}$ \\
\hline
\end{tabular}

${ }^{a} \mathrm{c}^{2}$ test. ${ }^{\mathrm{b}}$ Kaplan-Meier curves. ${ }^{\mathrm{c} I n d e p e n d e n t ~ s a m p l e ~ t-t e s t . ~ S M A, ~ s u p e r i o r ~ m e s e n t e r i c ~ a r t e r y ; ~} \mathrm{CA}_{19-9}$, cancer antigen 19-9; TBIL, total bilirubin; OS, overall survival; DFS, disease-free survival; LR, loco-regional recurrence; LM, liver metastasis; UICC, Union for International Cancer Control. 
A

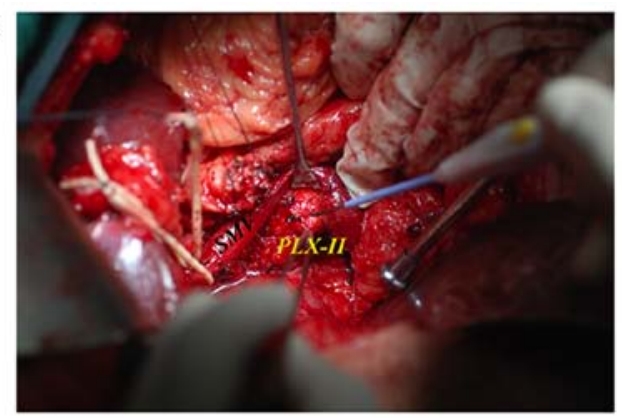

B

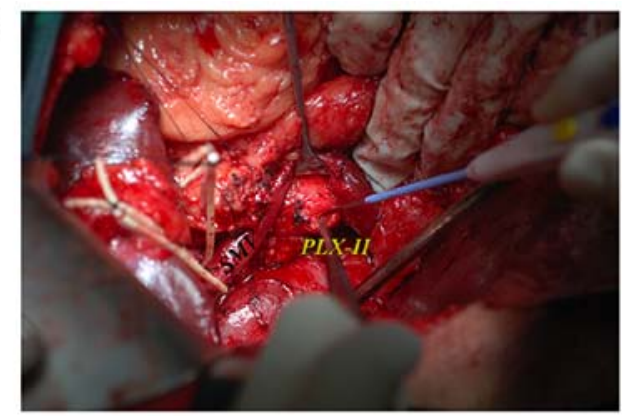

$\mathrm{C}$

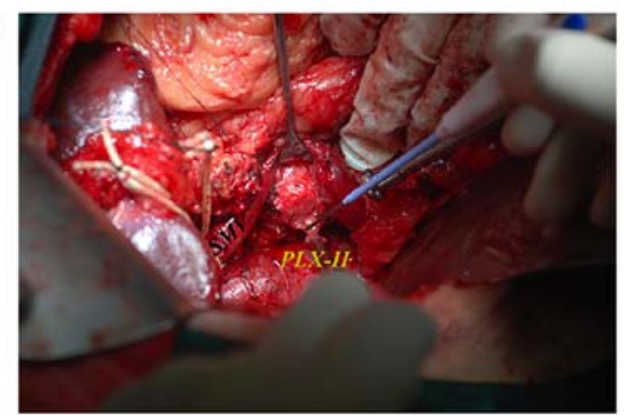

D

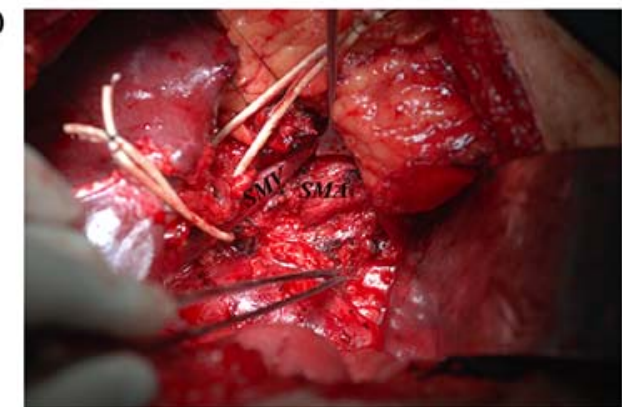

Figure 2. Process of obtaining the integrated tissues in the SMA margin during pancreaticoduodenectomy. (A) Exposed PLX-II region adjacent to the right-hand edge of the SMA. (B) Dissected PLX-II from the artery. (C) PLX-II was excised from the SMA. (D) Vascular surface of the SMA margin. SMA, superior mesenteric artery; SMV, superior mesenteric vein; PLX-II, pancreatic head plexus II.

$(\mathrm{P}=0.846)$. In addition, there was no association between the pathological characteristics, including IPNI $(\mathrm{P}=0.279)$, surgical margin $(\mathrm{P}=0.212)$, tumor differentiation $(\mathrm{P}=0.973)$ and $\mathrm{N}$ stage $(\mathrm{P}=0.556)$, and the SMA boundary. Venous invasion occurred predominantly in patients with obscure SMA boundaries $(\mathrm{P}=0.010)$, who were also more likely to have positive SMA margins $(\mathrm{P}<0.001)$ and poorer pathological UICC stages $(\mathrm{P}<0.001)$.

Survival analysis. The median follow-up time was 13 months; 121 cases of tumor recurrence were observed in $98(81.0 \%)$ patients at the final follow-up. The details of recurrence sites are summarized in Table II. For all patients, the mean OS was 14.4 months, the mean DFS was 11.1 months and the 1-year survival rate was $52.9 \%$ (Fig. 3). The univariate survival analysis of prognostic factors is summarized in Table III. The pattern of the SMA boundary on preoperative CE-CT $(\mathrm{P}=0.005)$, venous invasion $(\mathrm{P}<0.001)$ or resection $(\mathrm{P}=0.011)$, surgical margins $(\mathrm{P}=0.005)$, pathological $\mathrm{N}$ stage $(\mathrm{P}=0.015)$ and UICC stage $(\mathrm{P}=0.004)$ were all found to be significantly associated with OS. The prognostic factors for DFS included the SMA boundary $(\mathrm{P}=0.003)$, venous invasion or resection $(\mathrm{P}=0.001$ and $\mathrm{P}=0.027$ respectively), surgical margins $(\mathrm{P}=0.014)$ and UICC stage $(\mathrm{P}=0.019)$. Pathological T stage $(\mathrm{P}=0.021)$ and UICC stage $(\mathrm{P}=0.046)$ were associated with LR. The SMA boundary $(\mathrm{P}=0.001)$, venous invasion $(\mathrm{P}=0.004)$ and surgical margins $(\mathrm{P}=0.001)$ were risk factors for $\mathrm{LM}$.

The results of the multivariate survival analysis are presented in Table IV. The SMA boundary was independently associated with DFS $(\mathrm{P}=0.014)$ and LM $(\mathrm{P}=0.013)$. Patients with an obscure SMA boundary had a higher rate of recurrence and LM following radical resection, compared with those with a clear SMA boundary (Fig. 4A and B). Early recurrence occurred in $50 \%(34 / 68)$ of patients with clear
Table II. Sites of recurrence following radical surgery.

\begin{tabular}{lc}
\hline Sites of recurrence & No. of patients \\
\hline Loco-regional recurrence & 43 \\
Liver metastasis & 68 \\
Others & \\
Lung & 2 \\
Bone & 1 \\
Spleen & 1 \\
Peritoneum & 6 \\
Total & 21 \\
\hline
\end{tabular}

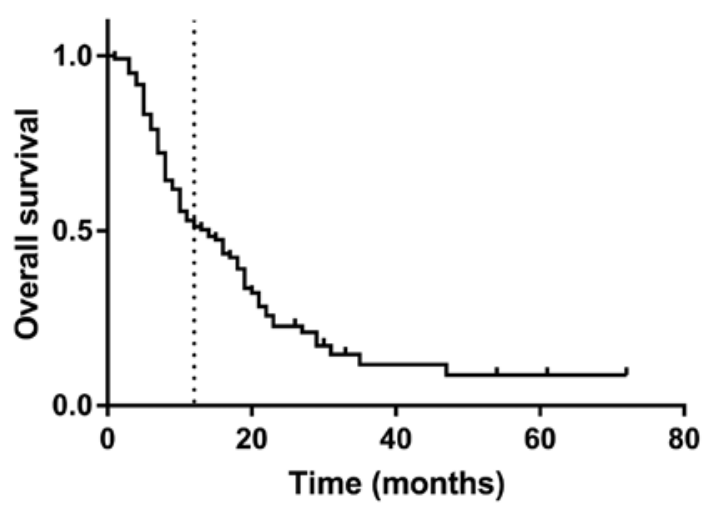

Figure 3. Kaplan-Meier curve of the overall survival in all patients. The 1-year survival rate was $52.9 \%$.

SMA boundaries and in $81.1 \%$ (43/53) of patients with obscure SMA boundaries $(\mathrm{P}<0.001)$. LM occurred within 12 months in 
Table III. Univariate survival analysis of prognostic factors.

\begin{tabular}{|c|c|c|c|c|}
\hline \multirow[b]{2}{*}{ Characteristic } & \multicolumn{4}{|c|}{ P-value } \\
\hline & OS & DFS & LR & LM \\
\hline Gender & 0.389 & 0.746 & 0.450 & 0.940 \\
\hline Age & 0.600 & 0.496 & 0.828 & 0.568 \\
\hline Venous invasion & 0.000 & 0.001 & 0.135 & 0.004 \\
\hline Venous resection & 0.011 & 0.027 & 0.301 & 0.309 \\
\hline SMA boundary & 0.005 & 0.003 & 0.182 & 0.001 \\
\hline $\mathrm{CA}_{19-9}$ & 0.615 & 0.528 & 0.570 & 0.739 \\
\hline TBIL & 0.501 & 0.619 & 0.308 & 0.937 \\
\hline Adjuvant therapy & 0.843 & 0.437 & 0.807 & 0.257 \\
\hline Surgical margin & 0.005 & 0.014 & 0.529 & 0.001 \\
\hline SMA margin & 0.184 & 0.211 & 0.611 & 0.067 \\
\hline Poor differentiation & 0.290 & 0.565 & 0.526 & 0.274 \\
\hline Cancer embolus in vessel & 0.577 & 0.342 & 0.627 & 0.977 \\
\hline Intrapancreatic neural invasion & 0.325 & 0.642 & 0.757 & 0.863 \\
\hline T stage & 0.139 & 0.158 & 0.021 & 0.520 \\
\hline $\mathrm{N}$ stage & 0.015 & 0.262 & 0.936 & 0.068 \\
\hline UICC stage & 0.004 & 0.019 & 0.046 & 0.146 \\
\hline
\end{tabular}

SMA, superior mesenteric artery; $\mathrm{CA}_{19-9}$, cancer antigen 19-9; TBIL, total bilirubin; T, tumor; N, node; UICC, Union for International Cancer Control; OS, overall survival; DFS, disease-free survival; LR, loco-regional recurrence; LM, liver metastasis.
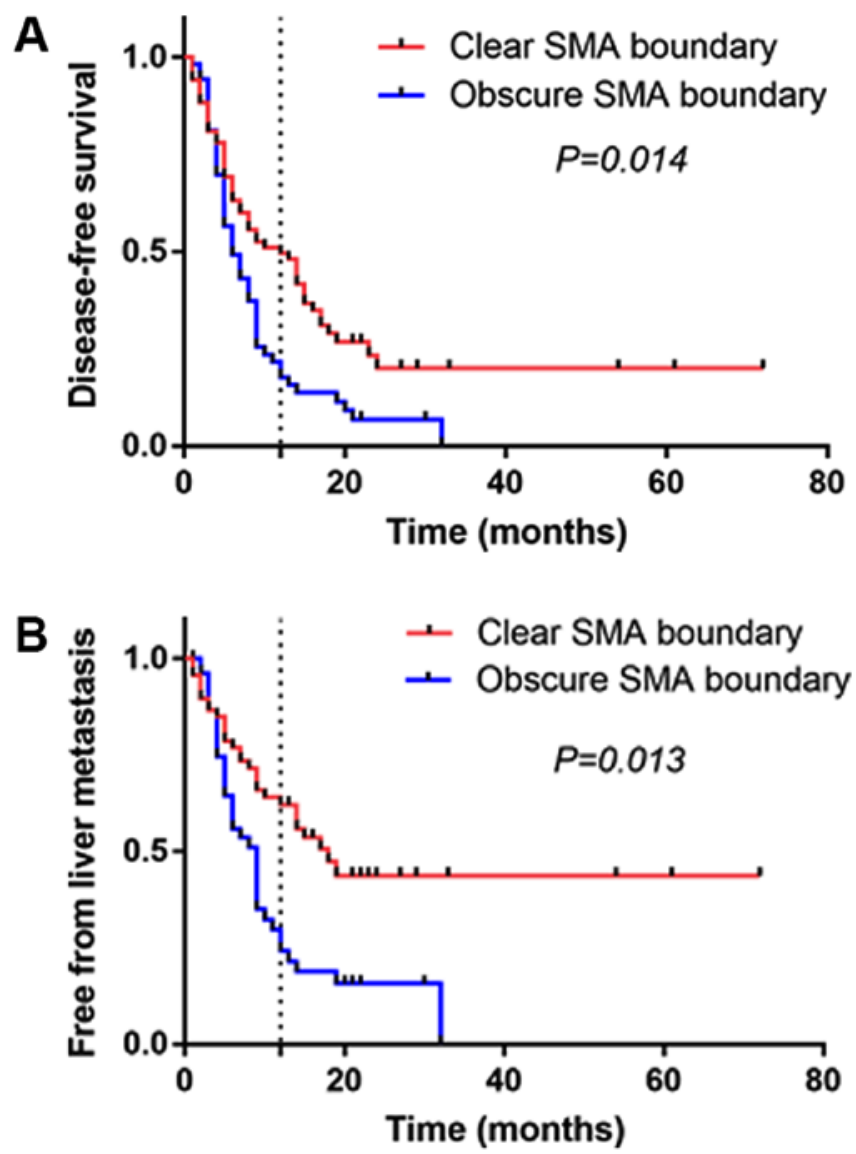

Figure 4. Association of DFS and LM with SMA boundary. Kaplan-Meier curves of (A) DFS and (B) LM among different SMA boundary groups. SMA, superior mesenteric artery; DFS, disease-free survival; LM, liver metastasis.
$33.8 \%(23 / 68)$ of patients with clear SMA boundaries, whereas the rate of LM was $64.2 \%(34 / 53)$ in patients with obscure SMA boundaries $(\mathrm{P}=0.001)$.

Pathological analysis of the SMA boundary. The pathological analysis of PLX-II revealed that extrapancreatic neural invasion (EPNI) occurred in $16.2 \%(11 / 68)$ of patients with clear SMA boundaries and in $13.2 \%(7 / 53)$ of patients with obscure SMA boundaries (Table V). Therefore, EPNI was not significantly associated with the pattern of the SMA boundary $(\mathrm{P}=0.649)$. VAChT-positive parasympathetic nerve fibers were observed in the SMA margins of all patients with pancreatic ductal adenocarcinoma, and the median number of parasympathetic nerve fibers was 24 (range, 7-55) per section. The number of parasympathetic nerve fibers in the SMA margin differed significantly between the two groups $(\mathrm{P}=0.014)$. Microscopic evaluation revealed that the parasympathetic nerve fibers were densely distributed in patients with obscure SMA boundaries, with a mean number of fibers was $30.2 \pm 11.5$ per section. By contrast, the mean number of such fibers in patients with clear SMA boundaries was $16.1 \pm 12.8$ per section, and these parasympathetic nerve fibers were sparsely distributed (Fig. 5A and B).

\section{Discussion}

Pancreatic head adenocarcinoma is characterized by early recurrence following radical resection and the guidelines that define borderline resectable pancreatic cancer are used to identify those patients who would not benefit from first-line surgery (5). However, even patients with resectable pancreatic 
Table IV. Multivariate survival analysis of prognostic factors.

\begin{tabular}{|c|c|c|c|c|c|}
\hline Characteristic & Variable & $\mathrm{n}$ & HR & $95 \% \mathrm{CI}$ & P-value \\
\hline \multicolumn{6}{|l|}{ Overall survival } \\
\hline \multirow[t]{3}{*}{$\mathrm{N}$ stage } & NO & 56 & 1 & & \\
\hline & $\mathrm{N} 1$ & 51 & 1.283 & $0.805-2.046$ & 0.295 \\
\hline & $\mathrm{N} 2$ & 14 & 2.507 & $1.280-4.910$ & 0.007 \\
\hline \multirow[t]{2}{*}{ Venous invasion } & No & 75 & 1 & & \\
\hline & Yes & 46 & 2.106 & $1.331-3.333$ & 0.001 \\
\hline \multicolumn{6}{|c|}{ Disease-free survival } \\
\hline \multirow[t]{2}{*}{ SMA boundary } & Clear & 68 & 1 & & \\
\hline & Obscure & 53 & 1.664 & $1.109-2.496$ & 0.014 \\
\hline \multirow[t]{2}{*}{ Surgical margin } & R0 & 58 & 1 & & \\
\hline & $\mathrm{R} 1$ & 63 & 1.539 & $1.024-2.313$ & 0.038 \\
\hline \multirow[t]{2}{*}{ Venous resection } & No & 101 & 1 & & \\
\hline & Yes & 20 & 1.828 & $1.067-3.131$ & 0.028 \\
\hline \multicolumn{6}{|l|}{ Liver metastasis } \\
\hline \multirow[t]{2}{*}{ SMA boundary } & Clear & 68 & 1 & & \\
\hline & Obscure & 53 & 1.878 & $1.142-3.087$ & 0.013 \\
\hline \multirow[t]{2}{*}{ Surgical margin } & No & 58 & 1 & & \\
\hline & Yes & 63 & 1.847 & $1.108-3.078$ & 0.019 \\
\hline
\end{tabular}

No characteristics were associated with loco-regional recurrence in multivariate analysis. SMA, superior mesenteric artery; HR, hazard ratio; $\mathrm{CI}$, confidence intervals.

Table V. Pathological characteristics of extrapancreatic plexus among patients with different SMA boundaries.

\begin{tabular}{lccc}
\hline Characteristic & Mean \pm SD/N $(\%)$ & $\begin{array}{c}\text { Clear SMA } \\
\text { boundary (n=68) }\end{array}$ & $\begin{array}{c}\text { Obscure SMA } \\
\text { boundary (n=53) }\end{array}$ \\
\hline $\begin{array}{l}\text { Parasympathetic nerves } \\
\text { (number of fibers/section) }\end{array}$ & $23.2 \pm 14.1$ & $16.1 \pm 12.8$ & $30.2 \pm 11.5$ \\
\begin{tabular}{l} 
Extrapancreatic neural invasion \\
\hline
\end{tabular} & $18(14.9)$ & $11(16.2)$ & $7(13.2)$ \\
\hline
\end{tabular}

${ }^{\mathrm{a}}$ Independent sample t-test. ${ }^{\mathrm{b}} \chi^{2}$ test. SMA, superior mesenteric artery.

head adenocarcinoma may succumb to early local recurrence or LM following first-line surgery (4). Previous research has revealed that, among patients with early (within 6 months) recurrence following surgery, the median survival time is poor (8-9 months) and the 5-year survival rate is $0 \%$ (20); however, the mechanism underlying early recurrence and metastasis remains to be fully elucidated.

All patients included in the present study had resectable tumors that did not appear to invade the main arteries on preoperative abdominal CE-CT and, thus, received standard pancreaticoduodenectomy. The pattern of the SMA boundary on CE-CT revealed the potential in indicating the prognosis of patients with resectable pancreatic head adenocarcinoma; patients with obscure SMA boundaries presented with poorer prognosis than patients with clear SMA boundaries. The SMA boundary was an independent prognostic factor of DFS $(\mathrm{P}=0.014)$ and $\mathrm{LM}(\mathrm{P}=0.013)$ following radical surgery. Early recurrence within 12 months (all recurrence sites) occurred in
$50.0 \%$ of patients with clear SMA boundaries, with a median DFS of 22.1 \pm 3.6 months; by contrast, early recurrence occurred in $81.1 \%$ of patients with obscure SMA boundaries and the median DFS was only 9.0 \pm 1.1 months. Early LM occurred in $33.8 \%$ of patients with clear SMA boundaries and $64.2 \%$ of patients with obscure SMA boundaries, with a median LM-free duration of $36.5 \pm 4.6$ and $11.2 \pm 1.5$ months, respectively. This suggests that the pattern of the SMA boundary reflected the biological behavior of the pancreatic head adenocarcinoma, with an obscure SMA boundary on CE-CT appearing to be associated with a higher degree of malignancy.

The obscure SMA boundary in CE-CT may be a radiological manifestation of EPNI (13-15). However, the results of the present study revealed that the obscure SMA boundary had no statistically significant association with neural invasion of the PLX-II located at the right-hand side of the SMA $(\mathrm{P}=0.649)$. Notably, previous studies have revealed tumor-nerve interactions in pancreatic cancer $(21,22)$. The influence of tumor cells on nerves 

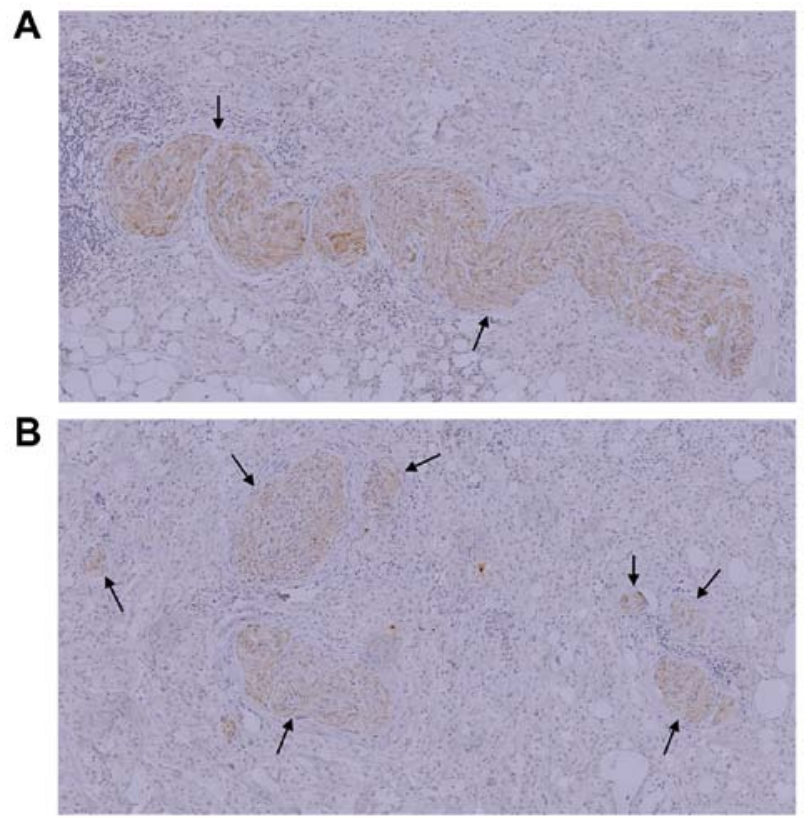

Figure 5. Representative photomicrographs showing staining of parasympathetic nerve fibers of the PLX-II. The black arrows indicate the parasympathetic nerve fibers stained by immunohistochemistry (magnification, x100). (A) Sparse distribution of parasympathetic nerve fibers (B) Dense distribution of parasympathetic nerve fibers. SMA, superior mesenteric artery; PLX-II, pancreatic head plexus II.

may lead to neuropathies, including sympathetic or parasympathetic neurogenesis $(23,24)$, neuritis $(25)$, nerve hypertrophy (26) or neural invasion (27). Similarly, neuropathy may also serve a key role in pancreatic neoplastic transformation, including tumor budding, which may occur prior to tumor invasion (28-30). Therefore, parasympathetic neurogenesis is a neuropathy that occurs in the tumor microenvironment (23). Immunohistological staining demonstrated that the pattern of the SMA boundary was associated with the grade of parasympathetic neurogenesis in the SMA margin $(\mathrm{P}=0.014)$; patients with obscure SMA boundaries exhibited more parasympathetic nerve fibers $(30.2 \pm 11.5$ fibers/per section) in the SMA margin compared with those with clear SMA boundaries (16.1 \pm 12.8 fibers/per section).

However, how the autonomic nervous system, including parasympathetic nerves, affects the prognosis of pancreatic head adenocarcinoma remains to be elucidated. Several studies have demonstrated that the peripheral nervous system may be considered as a neuronal circuit, which connects all organs to the central nervous system, and the internal organs may interact with the tumor microenvironment via the autonomic nerves (22). For example, sympathetic and parasympathetic nerves are reportedly necessary throughout all phases of prostate cancer progression in mice (31). The autonomic nerves are also essential components of the tumor microenvironment; they can regulate tissue homeostasis and promote cancer growth or metastasis by interacting with the majority of internal organs (32). Therefore, the sympathetic nervous system serves an important role in pancreatic cancer, and intratumoral parasympathetic neurogenesis may also be associated with tumor budding $(23,33)$.

Sympathetic and parasympathetic nerves may regulate tumor growth via direct innervation and the release of neurotransmitters (33). Nerves infiltrate the tumor microenvironment and release neurotransmitters directly into the cancer cell environment to stimulate their survival, proliferation and ability to spread; in turn, tumor cells stimulate nerve outgrowth $(21,22,34)$. This may explain why obscure SMA boundaries were associated with a positive SMA margin $(\mathrm{P}<0.001)$; it was suggested that parasympathetic neurogenesis may allow tumor cells to infiltrate connective tissue, thereby resulting in a positive SMA margin. Although the actions of the sympathetic and parasympathetic nervous systems are classically in opposition, they are in fact complementary in cancer, where sympathetic nerves stimulate early tumorigenesis and parasympathetic nerves activate the late metastatic process $(22,35)$. Prostate tumors with parasympathetic cholinergic fiber infiltration have a tendency for dissemination, and the density of nerves has been found to be directly associated with tumor aggressiveness (31). An increasing body of preclinical evidence has demonstrated that the seeding of pancreatic cancer cells in distant organs often occurs even prior to tumor formation at the primary site (36). In the present study, the density of parasympathetic nerves was associated with the SMA boundary; patients with a high number of parasympathetic nerves were more likely to have an obscure SMA boundary on preoperative CE-CT, which is predictive of early LM.

Early metastasis is responsible for early mortality, thereby providing a rationale for the administration of systemic therapy to patients with resectable pancreatic head adenocarcinoma or early-stage disease (37). The theory of nerve-cancer interactions has led to the development of innovative anticancer therapies; denervation treatment, for example, has been found to slow down cancer progression (38). Therefore, there is a rationale for neoadjuvant therapy in this setting, including that for the early treatment of potential micrometastatic disease that is responsible for postoperative recurrence, although this requires further validation in well-designed randomized trials (39). Patients with obscure SMA boundaries may develop early LM following radical surgery and may benefit from adjuvant hepatic arterial infusion chemotherapy following resection, which may effectively and safely prevent LM and improve prognosis (40); however, further investigation is required to confirm this hypothesis.

The present study had certain limitations. First, a number of patients were lost to follow-up, resulting in a median follow-up time of only 13 months. However, as the focus was on early recurrence (within 12 months), the results are considered accurate. Second, the present study only observed the association between parasympathetic neurogenesis in the SMA margin and the pattern of the SMA boundary, whereas other types of extrapancreatic neuropathy were not investigated. Finally, the present study was conducted in a single institution, and it was not population-based.

In conclusion, resectable pancreatic head adenocarcinoma is associated with a high risk for early relapse following surgery due to the intrinsic biological characteristics of the tumor. This suggests that first-line surgery is the optimal approach for only a minority of the patients. The SMA boundary on preoperative CE-CT may be associated with extrapancreatic neuropathies, including parasympathetic neurogenesis; an obscure SMA boundary may be a consequence of high-grade parasympathetic neurogenesis, and was identified as a predictive factor of a higher risk of recurrence and LM. Therefore, high-grade parasympathetic neurogenesis in the extrapancreatic plexus may be associated with a more aggressive phenotype of pancreatic 
head adenocarcinoma, and may assist in stratifying patients into prognostic subgroups to support surgeons in determining the optimal therapeutic strategies for individual patients.

\section{Acknowledgements}

Not applicable.

\section{Funding}

This study was supported by a grant from National Natural Science Foundation of China (grant no. 81672862) and the Capital Characteristic Clinical Application Research and Achievement Promotion Project (grant no.Z171100001017121).

\section{Availability of data and materials}

The datasets used and analyzed during the present study are available from the corresponding author on reasonable request.

\section{Authors' contributions}

DX, CY and ML conceived and designed the present study. LZ and LG performed the HE staining and immunohistochemistry. LG analyzed the results of the immunohistochemical experiment. ML, CY and LT performed statistical analysis and data interpretation; YP, ML and GL performed clinical data collection and samples collection; ML, CY and DX wrote the manuscript. All authors read and approved the final manuscript.

\section{Ethics approval and consent to participate}

The present study was exempted of informed consent. This study was approved by the Institutional Review Board of Peking University Third Hospital.

\section{Patient consent for publication}

Informed consent was waived in the present study, as all identifying information was removed, public interest considerations outweigh the potential harm, it was impossible to obtain permission and a reasonable individual would be unlikely to object to publication.

\section{Competing interests}

The authors declare that they have no competing interests.

\section{References}

1. Nipp RD, Zanconato A, Zheng H, Ferrone CR, Lillemoe KD, Wo JY, Hong TS, Clark JW, Ryan DP and Fernández-del Castillo C: Predictors of early mortality after surgical resection of pancreatic adenocarcinoma in the era of neoadjuvant treatment. Pancreas 46: 183-189, 2017.

2. Liu X, Xiu LJ, Jiao JP, Zhao J, Zhao Y, Lu Y, Shi J, Li YJ, Ye M, Gu YF, et al: Traditional Chinese medicine integrated with chemotherapy for stage IV non-surgical gastric cancer: A retrospective clinical analysis. J Integr Med 15: 469-475, 2017.

3. Shi H, Jin C and Fu D: Preoperative evaluation of pancreatic ductal adenocarcinoma with synchronous liver metastasis: Diagnosis and assessment of unresectability. World J Gastroentero 22: 10024-10037, 2016.
4. Stark AP, Sacks GD, Rochefort MM, Donahue TR, Reber HA, Tomlinson JS, Dawson DW, Eibl G and Hines OJ: Long-term survival in patients with pancreatic ductal adenocarcinoma. Surgery 159: 1520-1527, 2016.

5. Isaji S, Mizuno S, Windsor JA, Bassi C, Fernández-Del Castillo C, Hackert T, Hayasaki A, Katz MHG, Kim SW, Kishiwada M, et al: International consensus on definition and criteria of borderline resectable pancreatic ductal adenocarcinoma 2017. Pancreatology 18: 2-11, 2018.

6. Zaky AM, Wolfgang CL, Weiss MJ, Javed AA, Fishman EK and Zaheer A: Tumor-Vessel relationships in pancreatic ductal adenocarcinoma at multidetector CT: Different classification systems and their influence on treatment planning. Radiographics 37: 93-112, 2017.

7. Liu L, Katz MH, Lee SM, Fischer LK, Prakash L, Parker N, Wang H, Varadhachary GR, Wolff RA, Lee JE, et al: superior mesenteric artery margin of posttherapy pancreaticoduodenectomy and prognosis in patients with pancreatic ductal adenocarcinoma. Am J Surg Pathol 39: 1395-1403, 2015.

8. Denbo JW and Fleming JB: Definition and management of borderline resectable pancreatic cancer. Surg Clin North Am 96: 1337-1350, 2016.

9. Lopez NE, Prendergast C and Lowy AM: Borderline resectable pancreatic cancer: Definitions and management. World J Gastroentero 20: 10740-10751, 2014.

10. Bockhorn M, Uzunoglu FG, Adham M, Imrie C, Milicevic M, Sandberg AA, Asbun HJ, Bassi C, Büchler M, Charnley RM, et al: Borderline resectable pancreatic cancer: A consensus statement by the International Study Group of Pancreatic Surgery (ISGPS). Surgery 155: 977-988, 2014.

11. Peng Y, Xiu D, Jiang B, Ma Z, Yuan C, Su J, Shi X, Li L and Tao M: A clinical study about applying different R1 criteria to evaluate pancreatic head ductal adenocarcinoma specimens. Zhonghua Wai Ke Za Zhi 52: 834-838, 2014 (In Chinese).

12. Patel BN, Giacomini C, Jeffrey RB, Willmann JK and Olcott E: Three-dimensional volume-rendered multidetector CT imaging of the posterior inferior pancreaticoduodenal artery: Its anatomy and role in diagnosing extrapancreatic perineural invasion. Cancer Imaging 13: 580-590, 2013.

13. Noto M, Miwa K, Kitagawa $\mathrm{H}$, Kayahara M, Takamura $H$, Shimizu K and Ohta T: Pancreas head carcinoma: frequency of invasion to soft tissue adherent to the superior mesenteric artery. Am J Surg Pathol 29: 1056-1061, 2005.

14. Mochizuki K, Gabata T, Kozaka K, Hattori Y, Zen Y, Kitagawa H, Kayahara M, Ohta T and Matsui O: MDCT findings of extrapancreatic nerve plexus invasion by pancreas head carcinoma: Correlation with en bloc pathological specimens and diagnostic accuracy. Eur Radiol 20: 1757-1767, 2010.

15. Zuo HD, Tang W, Zhang XM, Zhao QH and Xiao B: CT and MR imaging patterns for pancreatic carcinoma invading the extrapancreatic neural plexus (Part II): Imaging of pancreatic carcinoma nerve invasion. World J Radiol 4: 13-20, 2012.

16. Bonnichon P, Rossat-Mignod JC, Corlieu P, Aaron C, Yandza T and Chapuis Y: Surgical approach to the superior mesenteric artery by the Kocher Maneuver: Anatomy study and clinical applications. Ann Vasc Surg 1: 505-508, 1987.

17. Shen SJ, Zhang YH, Gu XX, Jiang SJ and Xu LJ: Yangfei Kongliu Formula, a compound Chinese herbal medicine, combined with cisplatin, inhibits growth of lung cancer cells through transforming growth factor- $\beta 1$ signaling pathway. J Integr Med 15: 242-251, 2017.

18. StrobelO,Hank T,Hinz U,Bergmann F, SchneiderL,Springfeld C, Jäger D, Schirmacher P, Hackert T and Büchler MW: Pancreatic cancer surgery: The new R-status counts. Ann Surg 265: 565-573, 2017.

19. Allen PJ, Kuk D, Castillo CF, Basturk O, Wolfgang CL, Cameron JL, Lillemoe KD, Ferrone CR, Morales-Oyarvide V, He J, et al: Multi-institutional validation study of the American Joint Commission on Cancer (8th edition) Changes for $\mathrm{T}$ and $\mathrm{N}$ staging in patients with pancreatic adenocarcinoma. Ann Surg 265: 185-191, 2017.

20. Matsumoto I, Murakami Y, Shinzeki M, Asari S, Goto T, Tani M, Motoi F, Uemura K, Sho M, Satoi S, et al: Proposed preoperative risk factors for early recurrence in patients with resectable pancreatic ductal adenocarcinoma after surgical resection: A multi-center retrospective study. Pancreatology 15: 674-680, 2015.

21. Demir IE, Friess H and Ceyhan GO: Nerve-cancer interactions in the stromal biology of pancreatic cancer. Front Physiol 3: 97, 2012. 
22. Jobling P, Pundavela J, Oliveira SM, Roselli S, Walker MM and Hondermarck H: Nerve-cancer cell cross-talk: A novel promoter of tumor progression. Cancer Res 75: 1777-1781, 2015.

23. Zhang L, Guo L, Tao M, Fu W and Xiu D: Parasympathetic neurogenesis is strongly associated with tumor budding and correlates with an adverse prognosis in pancreatic ductal adenocarcinoma. Chin J Cancer Res 28: 180-186, 2016.

24. Demir IE, Friess H and Ceyhan GO: Neural plasticity in pancreatitis and pancreatic cancer. Nat Rev Gastroenterol Hepatol 12 : 649-659, 2015.

25. Ceyhan GO, Bergmann F, Kadihasanoglu M, Altintas B, Demir IE, Hinz U, Müller MW, Giese T, Büchler MW, Giese NA and Friess H: Pancreatic neuropathy and neuropathic pain-a comprehensive pathomorphological study of 546 cases. Gastroenterology 136: 177-186, 2009.

26. Ceyhan GO, Demir IE, Rauch U, Bergmann F, Müller MW, Büchler MW, Friess H and Schäfer K: Pancreatic neuropathy results in 'neural remodeling' and altered pancreatic innervation in chronic pancreatitis and pancreatic cancer. Am J Gastroenterol 104: 2555-2565, 2009.

27. Mitsunaga S, Hasebe T, Kinoshita T, Konishi M, Takahashi S, Gotohda N, Nakagohri T and Ochiai A: Detail histologic analysis of nerve plexus invasion in invasive ductal carcinoma of the pancreas and its prognostic impact. Am J Surg Pathol 31: 1636-1644, 2007.

28. Chouat E, Zehani A, Chelly I, Njima M, Maghrebi H, Bani MA Njim L, Zakhama A, Haouet S and Kchir N: Tumor budding is a prognostic factor linked to epithelial mesenchymal transition in pancreatic ductal adenocarcinoma. Study report and literature review. Pancreatology 18: 79-84, 2018.

29. Ceyhan GO, Demir IE, Maak M and Friess H: Fate of nerves in chronic pancreatitis: Neural remodeling and pancreatic neuropathy. Best Pract Res Clin Gastroenterol 24: 311-322, 2010.

30. O'Connor K, Li-Chang HH, Kalloger SE, Peixoto RD, Webber DL, Owen DA, Driman DK, Kirsch R, Serra S, Scudamore $\mathrm{CH}$, et al: Tumor budding is an independent adverse prognostic factor in pancreatic ductal adenocarcinoma. Am J Surg Pathol 39: 472-478, 2015.

31. Magnon C, Hall SJ, Lin J, Xue X, Gerber L, Freedland SJ and Frenette PS: Autonomic nerve development contributes to prostate cancer progression. Science 341: 1236361, 2013.
32. Demir IE, Ceyhan GO, Rauch U, Altintas B, Klotz M, Muller MW, Büchler MW, Friess H and Schäfer KH: The microenvironment in chronic pancreatitis and pancreatic cancer induces neuronal plasticity. Neurogastroenterol Motil 22: 480-490, e112-e113, 2010.

33. Guo K, Ma Q, Li J, Wang Z, Shan T, Li W, Xu Q and Xie K: Interaction of the sympathetic nerve with pancreatic cancer cells promotes perineural invasion through the activation of STAT3 signaling. Mol Cancer Ther 12: 264-273, 2013.

34. Kiba T: Relationships between the autonomic nervous system and the pancreas including regulation of regeneration and apoptosis: Recent developments. Pancreas 29: e51-e58, 2004.

35. Stopczynski RE, Normolle DP, Hartman DJ, Ying $H$ DeBerry JJ, Bielefeldt K, Rhim AD, DePinho RA, Albers KM and Davis BM: Neuroplastic changes occur early in the development of pancreatic ductal adenocarcinoma. Cancer Res 74: 1718-1727, 2014.

36. Sohal DP, Shrotriya S, Glass KT, Pelley RJ, McNamara MJ, Estfan B, Shapiro M, Wey J, Chalikonda S, Morris-Stiff G, et al: Predicting early mortality in resectable pancreatic adenocarcinoma: A cohort study. Cancer 121: 1779-1784, 2015.

37. Sinn M, Bahra M, Denecke T, Travis S, Pelzer U and Riess H: Perioperative treatment options in resectable pancreatic cancer-how to improve long-term survival. World J Gastrointest Oncol 8: 248-257, 2016.

38. Saloman JL, Albers KM, Li D, Hartman DJ, Crawford HC, Muha EA, Rhim AD and Davis BM: Ablation of sensory neurons in a genetic model of pancreatic ductal adenocarcinoma slows initiation and progression of cancer. Proc Natl Acad Sci USA 113: 3078-3083, 2016.

39. Khorana AA, Mangu PB, Berlin J, Engebretson A, Hong TS, Maitra A, Mohile SG, Mumber M, Schulick R, Shapiro M, et al: Potentially curable pancreatic cancer: American society of clinical oncology clinical practice guideline. J Clin Oncol 34: 2541-2556, 2016

40. Kurosaki I,Kawachi Y,NiheiK, Tsuchiya Y, Aono T, Yokoyama N, Shimizu T and Hatakeyama K: Liver perfusion chemotherapy with 5-Fluorouracil followed by systemic gemcitabine administration for resected pancreatic cancer: Preliminary results of a prospective phase 2 study. Pancreas 38: 161-167, 2009. 